\title{
The Use of Maxima in Teaching Analog and Digital Signal Processing
}

\author{
C. Gargour ${ }^{1}$, V. Ramachandran ${ }^{2}$ and M. Gabrea ${ }^{l}$ \\ 1.École de technologie supérieure (ETS), University of Québec, Montréal \\ 2. Concordia University, Montréal \\ christian.gargour@etsmtl.ca
}

\begin{abstract}
Maxima is mathematical software which can be obtained freely on the web. While Maxima it not specifically designed for analog and/or digital signal processing manipulations, its versatility and numerous symbolic as well as numerical manipulations capabilities makes it an interesting tool to be used by instructors and students of these types of courses. We will expose in this paper how Maxima can be used for that purpose and we will give numerous examples to illustrate its use in this domain.
\end{abstract}

Keywords : Maxima software, Analog signal processing, Digital signal processing.

\section{INTRODUCTION}

Maxima [1] is a publicly available open source software distributed under the GPL license [2]. Its origin is Macsyma, a computer algebra system which has been developed in the late 1960's at the Massachusetts Institute of Technology. Its source code can be compiled on Windows, Linux, and MacOS X. Precompiled binaries are also available. Maxima is a software capable of manipulating symbolic expressions: it can, among other things perform, differentiation, integration, solution of systems of linear and of ordinary differential equations, Taylor series expansions, Laplace transforms, etc. It has also the capability of handling vectors, matrices, polynomials, sets, lists, etc. Maxima can also manipulate numerical expressions in the form of fractions, arbitrary precision integers, and variable precision floating point numbers. It can be used to generate $2 \mathrm{D}$ and $3 \mathrm{D}$ plots of functions or data. A document based interface wxMaxima [3] for Maxima, distributed under the GPL license is also available.

The great flexibility of the Maxima software as well as the fact that it is free makes it an interesting tool for teaching purposes. It is our purpose to show in this paper how it can be fruitfully applied to the teaching analog and digital signal processing.

\section{ANALOG SIGNAL PROCESSING APPLICATIONS}

In this section we will give examples of application of Maxima to analog signal processing such as filter analysis and design etc.

It is quite easy it is to develop with Maxima simple sheets which can be easily modified in order to perform tasks commonly used in analog signal processing such as transforming one type of two-port parameters such as $\mathrm{Z}, \mathrm{Y}, \mathrm{H}$, etc. parameter into another type. An example of such use is given hereafter with the results provided by the software.

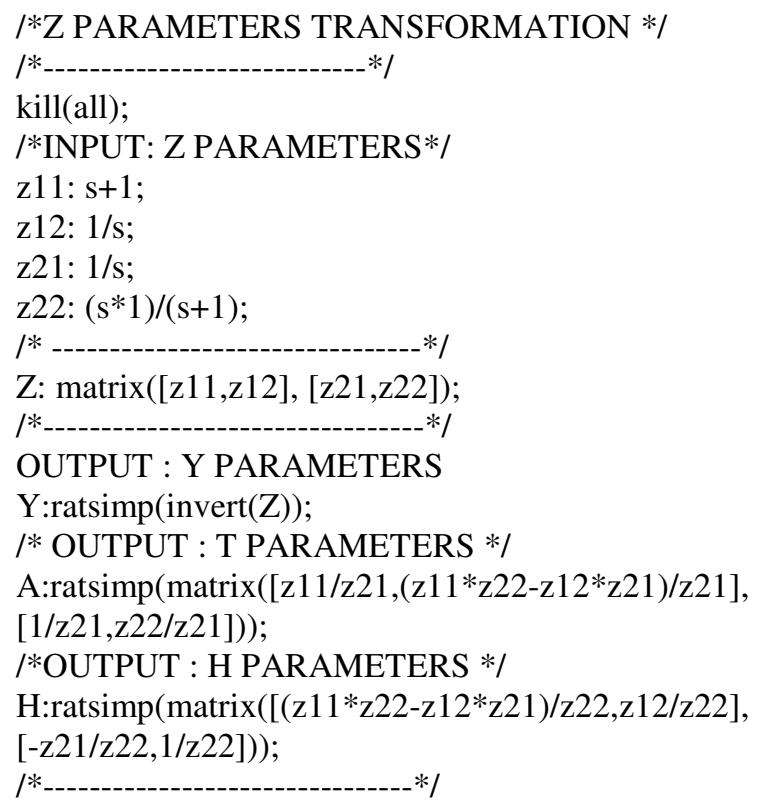




$$
\begin{aligned}
& \left(\frac{\circ}{8} 0\right) \text { done } \\
& \text { ( } \\
& \left(\frac{\circ}{8} \circ 2\right) \frac{1}{s} \\
& \text { (응 } \frac{1}{s} \\
& \left(\frac{8}{8} \circ 4\right) \frac{s}{s+1} \\
& \left(\frac{805)}{s+1}\left[\begin{array}{cc}
s+1 \\
\frac{1}{s} & \frac{s}{s+1}
\end{array}\right]\right. \\
& \left(\frac{\circ \circ)}{6}\left[\begin{array}{cc}
\frac{s^{3}}{s^{4}+s^{3}-s-1} & -\frac{s}{s^{3}-1} \\
-\frac{s}{s^{3}-1} & \frac{s^{3}+s^{2}}{s^{3}-1}
\end{array}\right]\right. \\
& \left(\frac{\circ \circ}{\partial} \circ\right)\left[\begin{array}{cc}
s^{2}+s & \frac{s^{3}-1}{s} \\
s & \frac{s^{2}}{s+1}
\end{array}\right] \\
& (\% \circ 8)\left[\begin{array}{cc}
\frac{s^{4}+s^{3}-s-1}{s^{3}} & \frac{s+1}{s^{2}} \\
-\frac{s+1}{s^{2}} & \frac{s+1}{s}
\end{array}\right]
\end{aligned}
$$

In this example we see how the symbolic manipulation capacities of Maxima are put to use.

Another example of the use of this software is the obtention of the minimum order $\mathrm{n}$ of an analog lowpass Butterworth filter fulfilling the following given specifications : maximum attenuation of $a \mathrm{~dB}$ at frequency $\Omega_{a}$ and minimum attenuation $A \mathrm{~dB}$ at $\Omega_{A}$ or of an analog low-pass Chebyshev filter fulfilling the following specifications : ripplewidth of $a \mathrm{~dB}$ in the range 0 to $\Omega_{a} \mathrm{rad} / \mathrm{s}$. and minimal attenuation of $A \mathrm{~dB}$ at $\Omega_{\mathrm{A}} \mathrm{rad} / \mathrm{sec}$.

A Maxima script performing this task is given hereafter.
/* BUTTERWORTH \& CHEBYCHEV ORDERS*/

/**/

kill (all);

/*INPUT PARAMETERS*/

a: 1 ;

wa: 1000 ;

A: 30 ;

wA: 2000;

/*BUTTERWORTH ORDER*/

$\mathrm{n}$ : float $\left(\left(\log \left(\operatorname{sqrt}\left(\left(10^{\wedge}(\mathrm{A} / 10)-1\right) /\left(10^{\wedge}(\mathrm{a} / 10)-\right.\right.\right.\right.\right.$

1))) $) / \log (w A / w a))$;

/*CHEBYSHEV ORDER*/

$\mathrm{n}$ : float $\left(\left(\operatorname{acosh}\left(\operatorname{sqrt}\left(\left(10^{\wedge}(\mathrm{A} / 10)-1\right) /\left(10^{\wedge}(\mathrm{a} / 10)-\right.\right.\right.\right.\right.$

1))))/acosh(wA/wa));

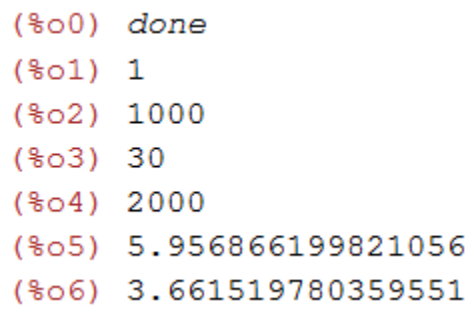

Here the answers have been left as non-integer quantities and should be rounded to the next integer which are respectively 6 for Butterworth and 4 for Chebyshev.

It is also possible to obtain expressions of the transfer functions of these filters by using Maxima

As well as utilizing its symbolic manipulations capacities to perform frequency transpositions to transform and/or designing bandpass, notch, etc. Filters starting from a prototype lowpass transfer function.

The following example shows how Maxima can be used to obtain the bandwidth $\Omega_{3 d B}$ of a number $n$ of number of identical finite gain noninverting amplifiers connected in cascade such that the total gain is $K_{P t o t}$ is specified as well as the gain bandwidth product $(G B)$ of each amplifier.

/*CASCADE CONNECTION OF AMPLIFIERS */

/* INPUT : SPECIFICATIONS */

kill(all);

GB: $10^{\wedge} 6$;

Kptot:200;

$\mathrm{W} 3 \mathrm{~dB}(\mathrm{n}):=\mathrm{GB}^{*}\left(\operatorname{sqrt}\left(2^{\wedge}(1 / \mathrm{n})-1\right)\right) /\left((\operatorname{Kptot})^{\wedge}(1 / \mathrm{n})+1\right) ;$ 
$\mathrm{n}:[1,2,3,4,5,6,7,8,9,10]$;

wxplot2d([discrete,n,W3dB(n)],[style,points]);

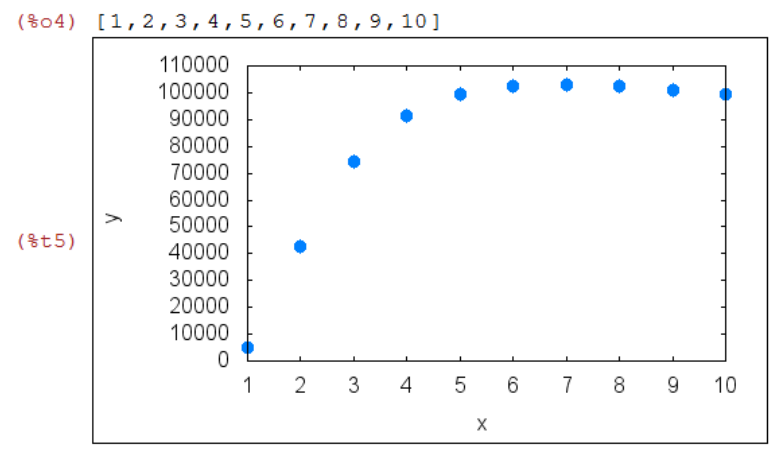

The highly non linear equation in $n$ which gives the system bandwidth is plotted for integer values on $n$ and the available bandwidth for each number of finite gain amplifier is available from the graph. It would also have been possible to use Maxima to solve this equation for the value of $n$ required for specified values of bandwidth $\Omega_{3 d B}$ and total gain $K_{P t o t}$.

A large number of other applications of the use of Maxima also exist and cannot be for obvious reasons of space availability, be given here.

\section{DIGITAL SIGNAL PROCESSING APPLICATIONS}

In this section we will give examples of application of Maxima to digital signal processing. We will start with the bilinear transformation. The following Maxima scripts apply the bilinear transformation to a $3^{\text {rd }}$ order bandpass filter. The $\mathrm{Z}$ transfer function is given by the software, then the magnitude and phase frequency responses of both the $S$-Domain transfer function and of the Z-Domain one are computed and plotted onthe same graphs for comparison purposes.

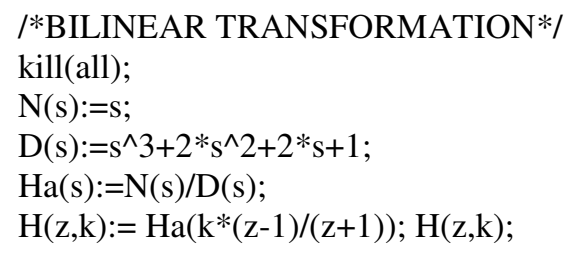

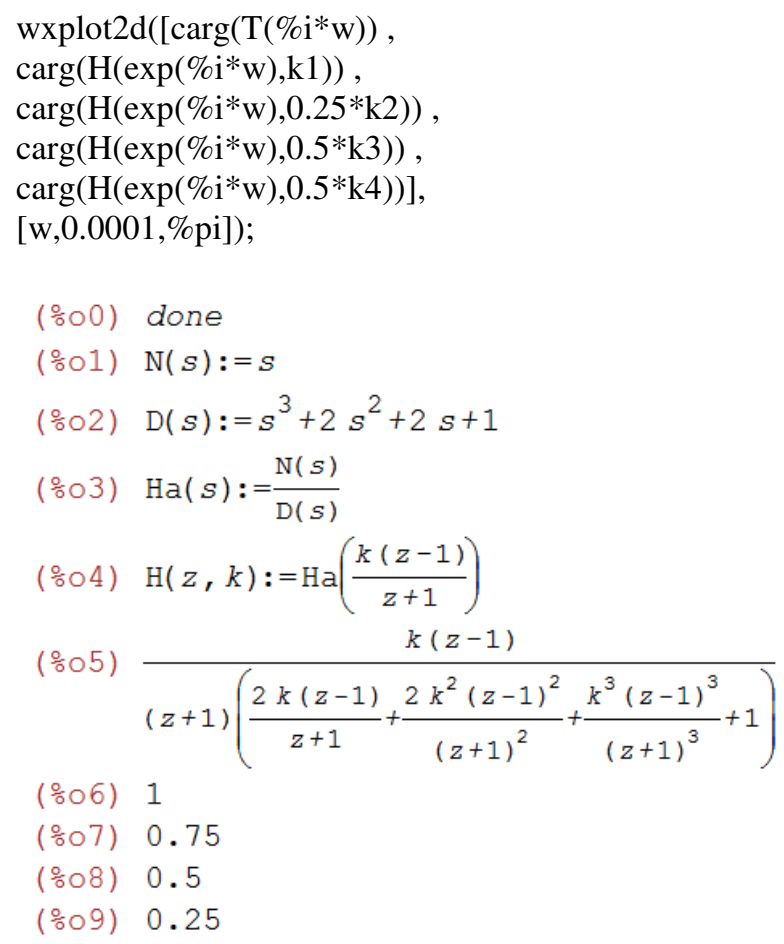
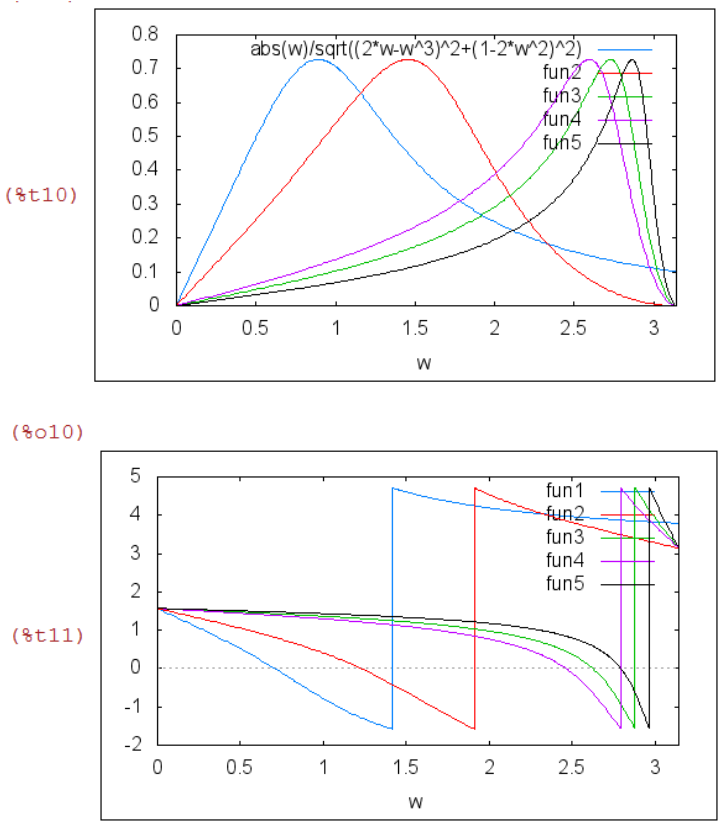

The following example's purpose is to obtain the frequency response (magnitude and phase) of a $7^{\text {th }}$, ordre comb filter.

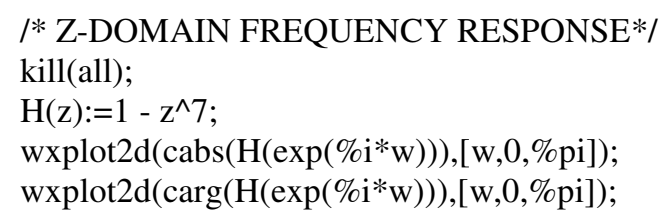



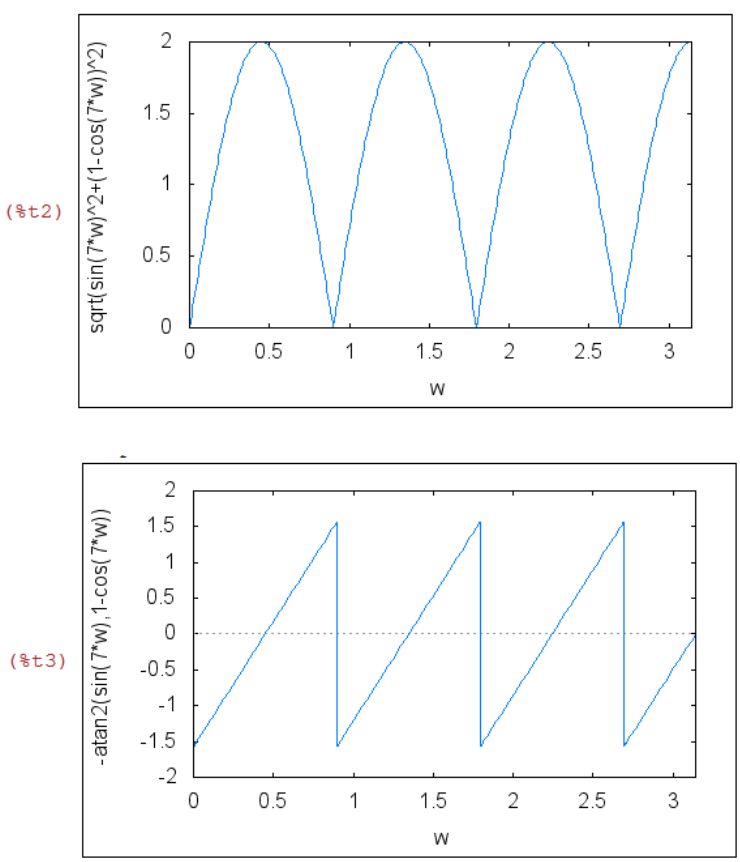

It is also possible to use Maxima to compute the Fourier transform of signals and windows, to solve analog and digital filter classical design problems, and much more.

\section{CONCLUSION}

As it has been illustrated in this paper It is quite easy it is to develop with Maxima simple scripts which can be easily modified in order to perform tasks commonly used in analog and digital signal processing with Maxima. This software is a general purpose mathematical tool which does not have specialized functions for signal processing. However this is not an inconvenient for teaching this subject. It could on the contrary be an advantage, because it leads to the use by the student of the basic principles to analyze and design analog and digital systems avoiding a " black box solution" approach which could result in a better understanding of the subject. Since this software is freely available it accessible to everybody
The capacity of Maxima to deal with both symbolic expressions and numerical data gives the student the possibility to manipulate the equations thus gaining insight to their behaviour. He can also obtain numerical solutions to the analysis and design problems which he tackles.

\section{References}

[1] http://maxima.sourceforge.net/ Maxima, a Computer Algebra System.

[2] http://www.gnu.org/copyleft/gpl.html GNU Operating System.

[3] http://andrejv.github.com/wxmaxima/ wxMaxima.

[4] http://www.csulb.edu/ woollett/ Maxima by Example, Edwin L. Woollett.

[5] http://maxima.sourceforge.net/docs/introm ax/intromax.html Introduction to Maxima by Richard Rand.

[6] http://math-blog.com/2007/06/04/a-10minute-tutorial-for-solving-math-problemswith-maxima/ A 10 minute tutorial for solving Math problems with Maxima by Antonio Cangiano.

[7]http://arachnoid.com/maxima/ Symbolic Mathematics Using Maxima, Paul Lutus.

[8] Digital Signal Processing, Principles, Algorithms, and Applications, $4^{\text {th }}$ ed. J.G. Proakis, D.G. Manolakis, Pearson, PrenticeHall, 2007.

[9] Discrete-Time Signal Processing A.V. Oppenheim, R.W. Schafer, Prentice-Hall, 2010.

[10] Digital Signal Processing, A Computer Based Approach, S.K. Mitra, McGraw Hill $4^{\text {th }}$. Ed. 2011. 